\title{
When people do not 'Zol': Reduced emergency centre attendance of patients with chronic obstructive pulmonary disease during coronavirus disease 2019 lockdown with the accompanying tobacco sales ban in South Africa
}

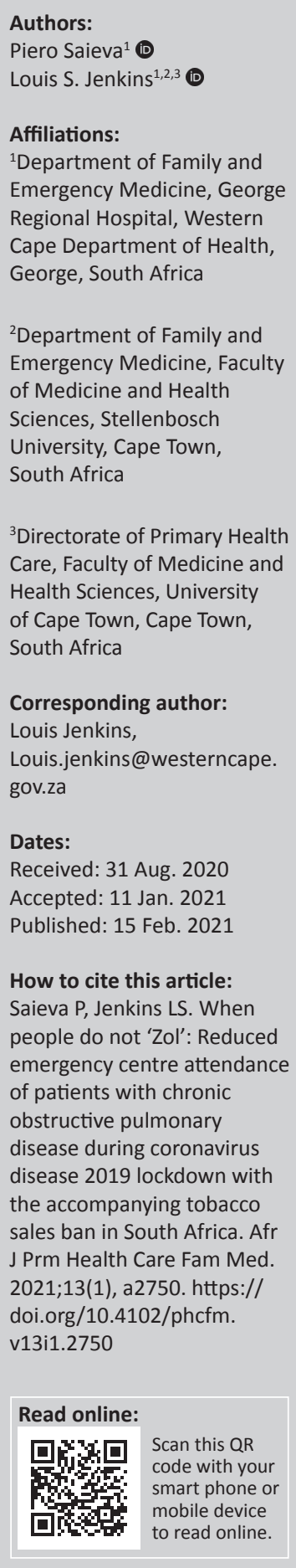

The coronavirus disease 2019 (COVID-19) pandemic has spread throughout the world, with devastating effects of the virus as well as the repercussions of the resulting 'lockdowns'. South Africa went into a national lockdown in March 2020 to mitigate the impact of the virus. This included a ban on the sales of tobacco and electronic cigarette products. The ban has been a highly contentious issue in South Africa, discussed worldwide, which has drawn many criticisms. The prevalence rate of smoking in South Africa was around 21.5\%, with the Western Cape province having a prevalence rate of $39 \%$. We compared the number of chronic obstructive pulmonary disease (COPD) presentations at a large regional referral hospital in the Western Cape province from January to August 2019 with the same period in 2020. Electronic emergency centre data showed a reduction of $69.28 \%$ in COPD presentations. To control for some confounders for the same period, we also reviewed patients presenting with urinary tract infections, which showed only a $30.60 \%$ reduction. This notable reduction in COPD presentations reduced service pressure of emergency centre and most likely benefitted patients' health. Further research and policies are needed to ensure ongoing reduction in the prevalence of smoking.

Keywords: smoking; restrictions; COVID-19; COPD; emergency reductions.

\section{Background}

Smoking is a common practice across Africa, with the estimated prevalence rate of $14.35 \%$ by the World Health Organization (WHO). ${ }^{1}$ It was previously suggested that 'Africa presents the greatest threat in terms of future smoking growth'. ${ }^{2}$ Cigarette smoking is known to be a major risk factor for developing and causing frequent exacerbations of chronic obstructive pulmonary disease (COPD). ${ }^{3}$ Chronic obstructive pulmonary disease has a prevalence rate of around $13.4 \%$ in Africa, with estimates of around $20 \%$ for South Africa (SA) ${ }^{4,5}$ Chronic obstructive pulmonary disease exacerbations have been linked to many triggers, such as change in season, air pollution, fires and smoking.

The coronavirus disease 2019 (COVID-19) pandemic was first reported in March 2020 in SA. During the same month, President Ramaphosa declared a national lockdown that restricted all services, except those that were deemed to be 'essential services'. ${ }^{6}$ Alcohol and cigarettes were classified as 'non-essential', which led to a ban on legal tobacco sales. ${ }^{6}$ Cigarette smoking is a common practice in SA, with an estimated national prevalence rate of $21.5 \%$ and a prevalence rate of $39 \%$ in the Western Cape Province. ${ }^{7}$ The role of smoking in altering the risk of developing COVID-19 remains unclear, although it appears to increase the disease severity. ${ }^{8}$ The reasons for the tobacco sales ban were multifactorial, according to Dlamini-Zuma, the South African Minister of Cooperative Governance and Traditional Affairs. She argued that smoking not only increases the severity of COVID-19 pneumonia but also increases the strain on the healthcare system, with more beds needed for COVID-19 patients as well as the usual emergencies. ${ }^{9}$ The ban was met with mixed reviews because of economic and personal factors, with one of the criticisms being the estimated daily loss of R31 million in excise tax in SA. ${ }^{10}$ 


\section{Methods}

\section{Setting}

George Regional Hospital is a 272-bed regional referral hospital in the Garden Route district of the Western Cape Province in SA. The emergency centre (EC) manages 4000 patients monthly, and with the surrounding primary healthcare clinics, cares for $84 \%$ of the local community of 210000 people.

\section{Sampling}

All patients attending the EC are entered into an online electronic patient information system called 'Hospital and Emergency centre tracking information system (HECTIS)' and receive a primary International Classification of Diseases ICD-10 diagnosis. Data on the total number of patients with COPD exacerbations attending from January to August in 2019 and for the same period in 2020 were extracted from HECTIS and analysed. We used the number of patients presenting with urinary tract infections (UTIs) during the same periods to control for possible confounders. January and February were included to show regular pre-lockdown figures for both the periods.

\section{Analysis}

Simple statistics, including frequencies and percentages, were calculated and entered in an Excel ${ }^{\mathrm{R}}$ spreadsheet.

\section{Ethical considerations}

This article followed all ethical standards for a research without direct contact with human or animal subjects.

\section{Results}

Compared with 2019, there was a significant drop in EC attendance of COPD patients during the same period in 2020.
In April 2020, there was a $75 \%$ (27 vs. 108) reduction compared with April 2019, in May 2020 a 70\% (35 vs. 166) reduction, in June $79 \%$ (23 vs. 109), in July 58\% (32 vs. 77) and in August there was a $61 \%$ (40 vs. 101) reduction. The combined reduction in COPD numbers during the year 2020 was $69.28 \%$ (see Figure 1).

With the national lockdown and the restrictions on movement, as well as the fear of accessing the hospital, we expected a decrease in patient numbers, as the hospital is the referral centre for severe COVID-19 infections in two districts. This was reflected in the HECTIS data that showed a reduction of patients presenting with UTIs, although not to the same extent as COPD numbers. In April 2020, there was a $27 \%$ (54 vs. 74 ) reduction in UTI cases, in May $46 \%$ (44 vs. 81 ), in June 39\% (39 vs. 64), in July 26\% (57 vs. 77) and in August $14 \%$ (60 vs. 70 ) reduction. The combined reduction in UTI numbers was $30.60 \%$ (see Figure 1).

\section{Discussion}

The main finding was a $69.28 \%$ reduction in patients presenting to the EC with COPD exacerbations, compared with that during the previous year for the same period. For the same period, there was a $30.60 \%$ reduction in patient numbers presenting to the EC for UTIs. Whilst both patient numbers with COPD and UTIs decreased during the lockdown period with the accompanying tobacco sales ban, it is noteworthy that COPD attendance was markedly more reduced. Whilst acknowledging various limitations because of possible confounders, such as the obligatory wearing of masks, curfew and other factors, one cannot ignore these observations.

Although SA was one of a few countries to introduce a tobacco sales ban during the COVID-19 lockdown (India and

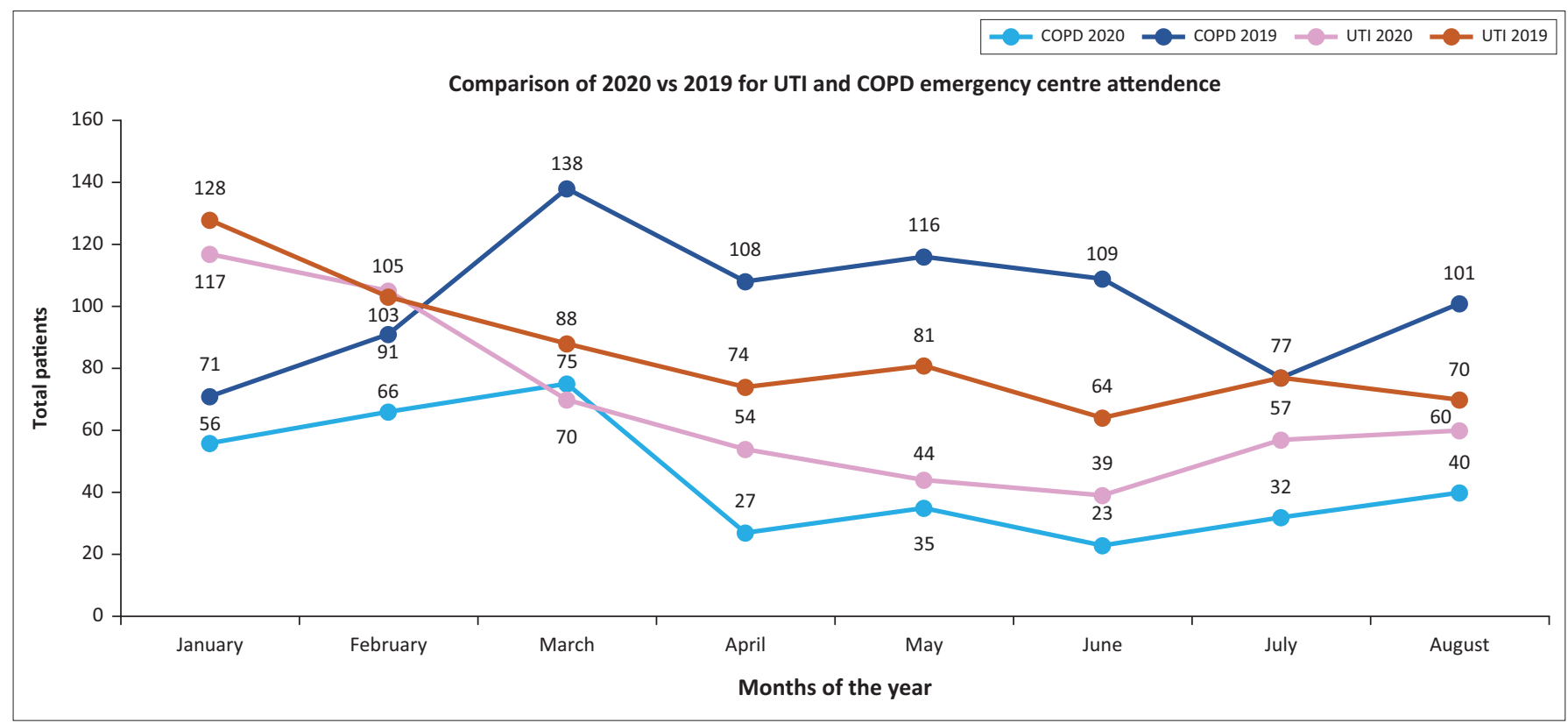

COPD, chronic obstructive pulmonary disease; UTI, urinary tract infection.

FIGURE 1: Comparison of patients presenting with chronic obstructive pulmonary disease and urinary tract infection at George Regional Hospital emergency centre. 
Botswana did the same), many countries have instituted measures to restrict smoking habits, with suggested concomitant reductions in diseases like COPD. ${ }^{11}$ Ireland instituted a national workplace smoking ban in 2004, with a $15 \%$ decrease in overall pulmonary admissions. ${ }^{12}$ A Canadian study in 2010, declaring public and work spaces smoke free, showed a $33 \%$ decrease in admissions of patients complaining with respiratory conditions (such as COPD, asthma, pneumonia and bronchitis). ${ }^{13}$ Whilst there is limited data from African studies, the findings from this small study correlate with international research into how tobacco sales restrictions have reduced COPD exacerbations and EC attendances.

A University of Cape Town survey found that during lockdown $90 \%$ of previous smokers bought cigarettes. ${ }^{14}$ These cigarettes were bought at extremely elevated prices, with an average 20 pack of cigarettes costing R144, whereas prior to the lockdown 20 packs of cigarettes costed between R25 and R45, on average. Single cigarettes were reported to cost R5.69, on average. There was a rise in unemployment because of the lockdown, with many households losing their primary source of income. It, therefore, seems likely that this unusual situation would have forced smokers to reduce the amount they are smoking. The survey showed that of the 12 118 participants, $42 \%$ had attempted to quit smoking. Whilst only $39 \%$ had succeeded, which is not unexpected, yet it is a step in the right direction. ${ }^{14}$

\section{Conclusion}

Whilst the tobacco sales ban has had detrimental effects on economy, one of the benefits has been a reduction in EC attendance of patients with COPD exacerbations, compared with that during the same period from the previous year. Whilst it is possible that the lockdown per se contributed to reduced EC attendance and reduced access to tobacco outlets or social gatherings, the net effect has been reduced pressure on the healthcare system because of a decreased number of patients attending the EC and needing admissions. We advocate for further research and policy into what confounders possibly played a role and which restrictions can be instituted to decrease the prevalence of smoking post lockdown, particularly in Africa.

\section{Acknowledgements}

The authors thank the George Regional Hospital management and the Emergency Centre healthcare workers for their support.

\section{Competing interests}

The authors have both declared that no competing interests exist.

\section{Authors' contribution}

P.S. conceptualised the article and carried out the data analysis. L.J. and P.S. contributed to subsequent drafts. Both the authors approved the final manuscript.

\section{Funding information}

This research received no specific grant from any funding agency in the public, commercial or not-for-profit sectors.

\section{Data availability}

Data are available upon request to the author.

\section{Disclaimer}

The views and opinions expressed in this research article are those of the authors and do not necessarily reflect the official policy or position of any affiliated agency of the authors.

\section{References}

1. World Health Organization. Report on global tobacco epidemic [homepage on the Internet]. World Health Organization, 2019 [cited 2020 Aug 26];157 p. Available from:https://apps.who.int/iris/bitstream/handle/10665/326043/9789241516204eng.pdf?ua $=1 \& u a=1$

2. Blecher $\mathrm{E}$, Ross $\mathrm{H}$. Tobacco use in Africa: Tobacco control through prevention. Atlanta, GA: American Cancer Society; 2013.

3. Burney $P$, Jithoo A, Kato B, et al. Chronic obstructive pulmonary disease mortality and prevalence: The associations with smoking and poverty-A bold analysis. Thorax. 2014;69(5):465-473. https://doi.org/10.1136/thoraxjnl-2013204460

4. Magitta F. Epidemiology and challenges of managing COPD in sub-Saharan Africa. Acta Sci Med Sci. 2018;2(1):17-23.

5. Viviers PJ, Van Zyl-Smit RN. Chronic obstructive pulmonary disease - Diagnosis and classification of severity. S Afr Med J. 2015;105(9):786-788. https://doi. org/10.7196/SAMJnew.8421

6. SA Government. Regulations and guidelines - Coronavirus COVID-19 [homepage on the Internet]. South African Government. [cited 2020 Jul 26]. Available from: https://www.gov.za/coronavirus/guidelines

7. National Department of Health (NDoH), Statistics South Africa (Stats SA), South African Medical Research Council (SAMRC), and ICF. South Africa Demographic and Health Survey 2016. Pretoria and Rockville, MA: NDoH, Stats SA, SAMRC, and ICF; 2019.

8. Igić R. Smoking and COVID-19. Vojnosanit Pregl. 2020;77(5):461-462. https://doi. org/10.2298/VSP2005461।

9. Mabuza E. Smoking ban a sign of a 'responsible government', says Dlamini-Zuma [homepage on the Internet]. Times Live. [cited 2020 Jul 26]. Available from: https://www.timeslive.co.za/politics/2020-06-04-smoking-ban-a-sign-of-aresponsible-government-says-dlamini-zuma/

10. Leech K. Up in smoke: Roughly R35m in tobacco tax revenues lost daily during South Africa's lockdown [homepage on the Internet]. Africacheck.org. 2020 [cited 2020 Jul 25]. p. 1. Available from: https://africacheck.org/reports/up-in-smokeroughly-r35-mil-in-tobacco-tax-revenues-lost-daily-during-south-africaslockdown/

11. Kahn T. WHO and scientists back SA's ban on tobacco sales [homepage on the Internet]. Business Day. [cited 2020 Dec 18]. Available from: https://www. businesslive.co.za/bd/national/health/2020-05-04-who-and-scientists-back-sasban-on-tobacco-sales/

12. Stallings-Smith S, Zeka A, Goodman P, Kabir Z, Clancy L. Reductions in cardiovascular, cerebrovascular, and respiratory mortality following the Nationa Irish smoking ban: Interrupted time-series analysis. PLoS One. 2013;8(4):e62063. https://doi.org/10.1371/journal.pone.0062063

13. Naiman A, Glazier RH, Moineddin R. Association of anti-smoking legislation with rates of hospital admission for cardiovascular and respiratory conditions. CMAJ. 2010;182(8):761-767. https://doi.org/10.1503/cmaj.091130

14. Van Walbeek C, Filby S, Van der Zee K. Lighting up the illicit market: Smoker's responses to the cigarette sales ban in South Africa [homepage on the Internet]. 2020 [cited 2020 Aug 26];1-43. Available from: http://www.reep.uct.ac.za/sites/ default/files/image_tool/images/405/Publications/reports/LockdownSurvey Final.pdf 